\section{Blutentnahme: Nüchtern nicht nötig}

Querschnittsuntersuchungen aus den USA analysierten bei 12.744 Kindern zwischen drei und 17 Jahren den Einfluss der Zeitdauer nach der Mahlzeit auf Gesamt-Cholesterin, HDL-, LDLund Triglyceridspiegel. Erstaunlicherweise führte eine längere Fastenperiode eher zu einem um 2-5 mg/dl höheren Gesamt-Cholesterin sowie höherem HDL- und LDL-Cholesterin. Die Triglyceride lagen zwar bis zu $7 \mathrm{mg} / \mathrm{dl}$ niedriger, was aber eine geringe Bedeutung hat. Das Ergebnis zeigt, dass eine Nüchternblutentnahme für die Analyse der Lipidprofile völlig unnötig ist. Pediatrics 2011, 3:463

\section{Prostatabiopsie: Gar nicht harmlos}

Nach der Stanzbiopsie erlitten 25 der 1000 Untersuchten einer US-Studie schwere Nachwirkungen. Darunter achtmal akuter Harnverhalt und viermal massive Hämaturien. Von diesen entwickelten drei einen Blutpfropf, einer glitt sogar in die Niereninsuffizienz ab. Rund die Hälfte der schweren Komplikationen waren trotz prophylaktischer Gabe von Ciprofloxacin oder Cotrimoxazol infektiöser Natur. In $92 \%$ der Fälle war E. coli der Verursacher und erwies sich immer als resistent gegen mindestens eines der präventiv verabreichten Anitibiotika. BJU international 2012 (online first)

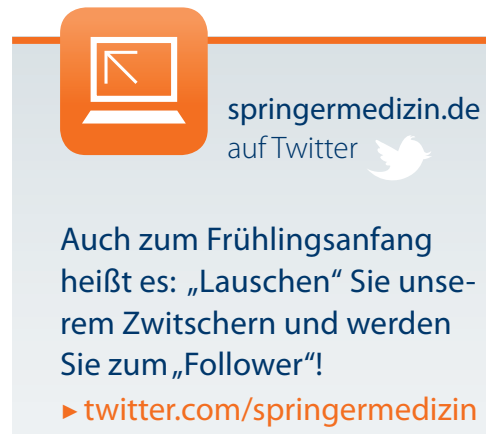

Fieser Feinstaub \& Co. Smog triggert Herzinfarkte

\section{Starke Luftverschmutzung greift die Gesundheit an - Mit Abgasen be- lastete Luft kann sogar Herzinfarkte auslösen. Fast alle Luftschadstoffe lassen bereits innerhalb kurzer Zeit das Herzinfarktrisiko kurzfristig an- steigen.}

Starke Luftverschmutzung greift die Gesundheit an. Mehr Menschen werden in ein Krankenhaus eingeliefert, mehr Menschen sterben an Atemwegsoder Herz-Kreislauf-Erkrankungen um nur einige der in epidemiologischen Studien beobachteten Folgen zu nennen. Die Studienlage ist jedoch widersprüchlich, was die kurzfristigen Auswirkung von Smog. In einer Metaanalyse haben Pariser Ärzte den Zusammenhang deshalb für die einzelnen Hauptschadstoffe aufgeschlüsselt. Die Auswertung von
34 Studien brachte ans Licht, dass außer Ozon alle untersuchten Luftschadstoffe das Risiko kurzfristig zunehmen ließen. Dabei stieg das Risiko bei Kohlenstoffmonoxid pro $1 \mathrm{mg} / \mathrm{mg}^{3} \mathrm{CO}$ um $4,8 \%$. Bei Stickstoffdioxid und Schwefeldioxid stieg das Herzinfarktrisiko pro $10 \mu \mathrm{g} / \mathrm{m}^{3}$ um $1,1 \%$ und $1,0 \%$ und bei Feinstaubpartikeln $\leq 10 \mu \mathrm{m}\left(\mathrm{PM}_{10}\right)$ und $\leq 2,5 \mu \mathrm{m}\left(\mathrm{PM}_{2,5}\right)$ pro $10 \mu \mathrm{g} / \mathrm{m}^{3}$ um $0,6 \%$ sowie $2,5 \%$.

\section{Weit verbreiteter Risikofaktor}

Damit ist die Schadwirkung von Smog zwar geringer als bei den klassischen Risikofaktoren. Trotzdem kann man den Beitrag auf Bevölkerungsebene nicht ignorieren, ist doch praktisch jeder Städter der Luftverschmutzung wahllos ausgesetzt.

(mg)

\section{Von Mundhöhle bis Kolon}

\section{Glas für Glas dem Krebs entgegen}

Schon ab einem Glas (ca. $10 \mathrm{~g}$ ) Alkohol pro Tag beginnt das Risiko für Krebserkrankungen in Mundhöhle, Rachen und Oesophagus zu steigen. Kolon und Pankreas sind alkoholresistenter - hier liegt die kritische Grenze bei vier Gläsern pro Tag.

Wie erwartet, hatten vor allem die starken Trinker schlechte Karten in Sachen Krebsrisiko. Das relative Risiko (RR) für Krebs in Mundhöhle und Pharynx liegt bei ihnen bei 5,24 (31 Studien), in Ösophagus bei 4,89 (39 Studien). In der italienisch-amerikanischen Untersuchung zeigten sich allerdings Unterschiede hin- sichtlich der verschiedenen Tumorlokalisationen. Das RR für Kolonkarzinome lag im Vergleich bei 1,52 (19 Studien) für Pankreas bei RR 1,22 (13 Studien).

\section{Schon ein Glas Wein pro Abend}

Aber auch ein Glas Alkohol erhöht das Risiko für Mundhöhle und Pharynx, verglichen mit Nicht- oder Gelegenheitstrinkern, immerhin um RR 1,21 (20 Studien). Würde Alkohol in den empfohlenen Maßen getrunken, könnten bei den Männern etwa $90 \%$ der alkoholbedingten Krebserkrankungen und bei Frauen über 50\% verhindert werden.

(st) 